\title{
Video Quality Analysis of Distributed Video Coding in Wireless Multimedia Sensor Networks
}

\author{
Mortaza Nikzad, Ali Bohlooli, Kamal Jamshidi \\ Faculty of Computer Engineering, University of Isfahan, Isfahan, Iran \\ $\{$ mrtz_azad, Bohlooli, Jamshidi\}@eng.ui.ac.ir
}

\begin{abstract}
Multimedia communications in wireless sensor networks is a very challenging task. Video coding with high computational complexity and great contribution to the energy consumption of nodes and video transmission over erroneous wireless channel are the main reasons of these challenges. Distributed Video Coding has high potential for being deployed in these networks due to its unique features like as independent frame coding and low complexity encoding operations. The purpose of this study is to understand and evaluate the distributed video coding performance facing the transmission characteristics in wireless multimedia sensor networks. To this end, the comparative analysis of the coding in respect of main factors of video transmission (i.e., bit rate and error resiliency) in the Wireless Multimedia Sensor Networks (WMSN) has been done. We have used both the objective and subjective criteria for checking the video quality and applied the Gilbert-Elliot channel model for capturing the bit-level error in WMSN. Therefore, unlike previous works, our results are more realistic. In addition, based on this model we have investigated the impact of protection of frames by Reed-Solomon error control scheme. The results show that paying attention to coding parameters and protecting key frames, have a great impact on increasing the quality of the receiving video and will reduce the energy consumption and delays due to low number of requests from the feedback channel.
\end{abstract}

Index Terms-Multimedia communications, Wireless Multimedia Sensor Networks, Distributed Video Coding.

\section{INTRODUCTION}

Wireless Multimedia Sensor Networks (WMSN) are new class of Wireless Sensor Networks that have high potential for solving the problems related to interaction with the real world in the industries, environments, medical, and security applications [1, 2]. Strict and predefined QOS requirements of multimedia data on a network of low-power nodes pose many challenges in respect of energy, ability to process and storage capacity. Now, among the several types of multimedia data, compression and transmission of video data on WMSN are the greatest challenges because in one hand, it has heavy process at encoding side, high bit rate, and strict quality requirements and on the other hand, we are faced with constraints arising from low power nodes and erroneous wireless channels that practically make the traditional video coding standards like MPEG or H.26x impossible for these networks [3, 4].

Distributed Video Coding is the best option for being used in WMSN because of low computational complexity of video coding $[4,5]$. In addition, independently frame coding in this codec that is because of its unique architecture, provides ability to multi view scenes without any need for communication between nodes in the case of dense networks. These features enable many other creative applications that are not possible for WMSN using conventional video coding [6].

But designing the efficient and high quality transmission frameworks for this coding are not possible without evaluating and understanding the DVC performance facing the transmission characteristics in WMSN. For this reason, in this study a series of comparative analyzes has been presented for the two main transmission factors: bit rate and error resiliency which are the most important properties of optimal transmission over WMSN [7].

Rest of this paper is organized as follows: Section 2 is dedicated to reviewing distributed video coding architecture and the related previous works. Then in Section 3, performance of bit rate is analyzed in this coding for all types of frames. Analysis of DVC error resilience is presented in Section 4 after describing the simulation conditions. Next, the effect of protection of key frames by RS error control on perceived quality has been investigated. Finally, Section 5 is dedicated to conclusion and future works.

\section{BACKGROUND}

Due to unique properties of DVC in recent years, many researchers focus on it $[6,8]$. This method of video coding does not mean distribution operations related to video coding between nodes; in fact this coding method is an independent way for video compression which is based on Wolf-Slepian theory in information theory that was presented as distributed source coding[9] for lossless separate coding in 1970s. Then, it was presented as a lossy coding by Wyner-Ziv[10], and it has been used for video coding in recent years.

Although, data coding and multimedia data experts look for more efficient architectural coding method [8], especially for presenting some architectures in order to support better quality for multi view feature in this coding [6], now two architectures are the most common ones for DVC [11]. The first architecture, interpolation based distributed video codec, was presented at Stanford University [12, 13] and shortly afterwards, the second architecture, a backward prediction based distributed codec that known as PRISM [14, 15], was presented. 
According to observations and researches, the first architecture has shown better performance than the second architecture due to bit rate [11]; therefore, we will consider the first architecture in this paper. Its block diagram is shown in Fig. 1.

Main idea in this coding method is to attempt to exploit the temporal correlation of video signal in the decoding phase rather than in the encoding one. For this reason, frames will be encoded completely independent of each other in this coding method.

In DVC, key frames are encoded by one of the traditional video codec like H.264/AVC in intra mode operation without any motion compensated prediction between two frames. Also inter frames known as WynerZiv frames are encoded based on Distributed Source Coding principles. To this end, after suitable DCT transformation and quantization operation thanks to proper quantization matrices, bits will be sent to a turbo encoder.

Turbo encoder is a systematic channel coding that produces parity bits by encoding the frames and sending them to decoder by discarding the main data. Also Turbo decoder uses them in decoder in order to full reconstruction of the $\mathrm{WZ}$ frame that its rough estimation was created by doing motion compensation interpolation in decoder, between two previous key frames by name of Side Information (SI) [11-13]. Then, since no prediction loop exists in encoder, an intrinsic robustness to transmission errors has been claimed. So, the complexity of coding operations will be transferred from encoder to decoder.

A notable point in this architecture is the existence of a feedback channel. As it is shown in Fig. 1, all external bits from turbo encoder are not sent to decoder and based on the balance between bit rate and quality of picture a suitable size of bits that are sent to the decoder should be approximated. However, in decoding time, if we are not able to reconstruct its corresponding WZ frames for any reason such as bad SI, we can request and receive more parity bits from feedback channel.

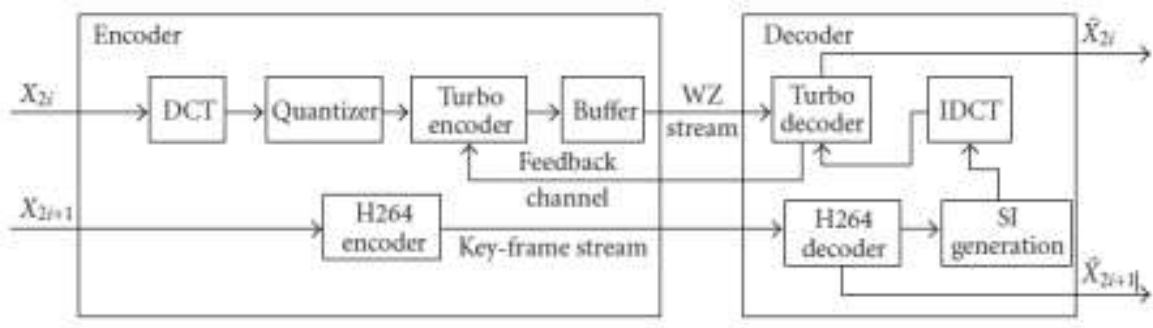

Fig. 1. Distributed Video Coding Architecture

So, the feedback channel and independent behavior of frames from each other are caused the basic difference between this coding method and the traditional video coding. For this reason, many researchers are looking for optimizing video transmission due to this coding method on computer networks. For example, in [16] has presented the cross layer framework for video transmission with high quality on wireless multimedia sensor networks that has used DVC coding in the application layer of this framework. But it only has sufficed to different prioritizing its frames in wireless medium access (control) layer that in fact is a common technique in transmission of video that coded with traditional video coding, too.

Tonoli et al. [11] observed error-resiliency in current architectures of this coding and analyzed the effect of error on video quality in this coding. But a suitable channel model has not been used in this work. They used packet level channel model that does not obtained valid results for video due to none equal video frame size and varies of error occurrence over time in the RF band that used in WMSN.

In this research, an appropriate error model is presented for channels that act in bit level. Then, video transmission from the distributed video coding is analyzed by considering the constraints posed by wireless sensor networks. Therefore, its results will be more realistic and it can be used in the frameworks of video transmission especially in cross layer video transmission framework which are based on the information of application layer.

\section{ANALYSIS OF BIT RATE EFFICIENCY}

Data transmission consumes a large amount of energy in sensors [1]; thus, in these networks relationship between perceived quality and bit rate for video coding has a double significance and a suitable video coding approach for these networks should have high performance on bit rate in addition to having the low complexity in the coding and intrinsic robustness to transmission error. In order to evaluate this criterion in the current architecture of DVC, in this section of the paper, at first we compare the bit rate performance of DVC and H264/AVC coding. Then, we analyze DVC operation in encoding with different bit rates in order to determine the effect of independently coded frames on the importance and efficiency of bit rates in key and Wyner-Ziv frames. To do this, two video sequences "Foreman" and "Hall monitor" that are varying in motion characteristics and widely used in papers, were selected. These video sequences contain 300 frames of QCIF resolution and they have coded with rate of 30 frames per second by DISCOVER [17].

In Fig. 2 and Fig. 3, rate-distortion curve for these two coding methods are observable in different GOP sizes. Here, distortion is based on luminance values in picture. 
In both of sequences, DVC has much higher efficiency than H264/AVC at intra mode. Also it is observed that, after a certain bit rate, DVC acts better than H264/AVC with GOP size 2. The reason is formed better SI from previous key frames due to higher bit rate and so, the decoder does not need to receive more parity bits.

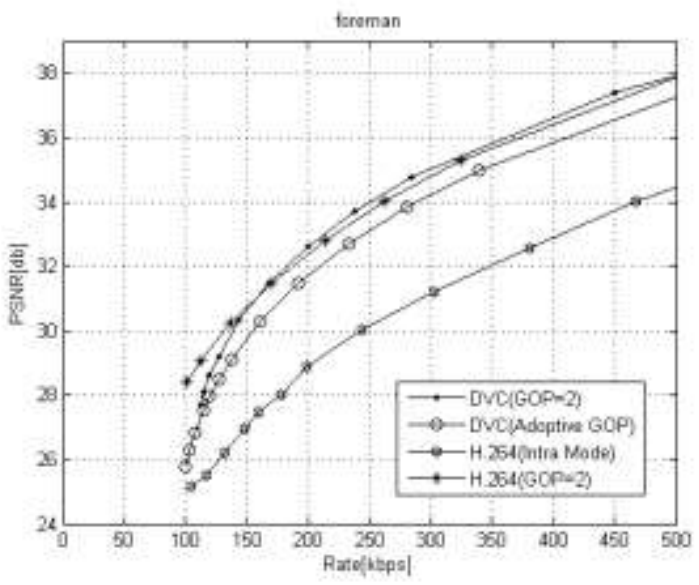

Fig. 2. Rate-Distortion comparison in DVC and H.264 in the Foreman sequence

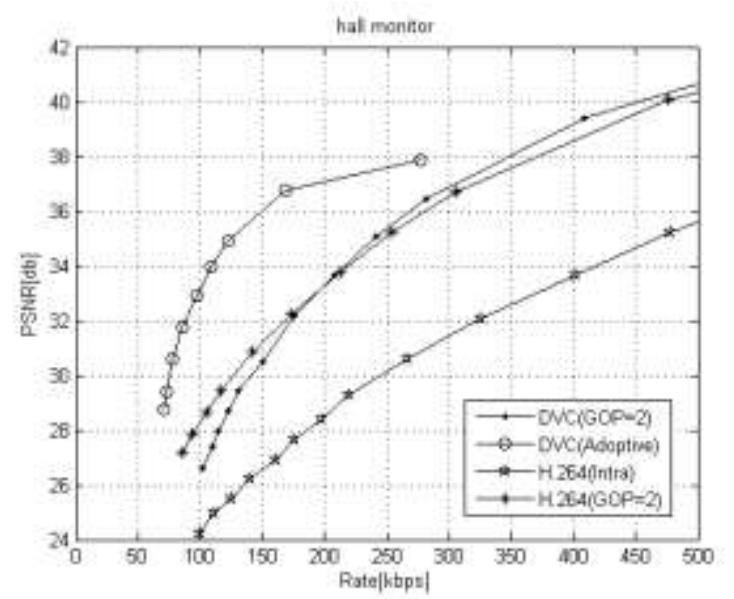

Fig. 3. Rate-Distortion comparison in DVC and H.264 in the Hall monitor sequence

Another remark in this diagram is higher bit rate of DVC with Adoptive GOP size from GOP size 2 and also, H264/AVC. In fact, it occurs because of high motion in frames and codec preference on sending frames completely instead of sending WZ frames. This issue is inversed in Fig. 2 that has obtained for video with low motion and as you see, DVC has achieved high performance on bit rate with succession sending of several WZ frames.

An important note which should be considered about this coding is carefully choosing the parameters of the coding, especially, quantization parameter and consequently the bit rate. In fact, since size of required parity bits in this coding that has been requested by decoder for reconstruction related WZ frames is dependent on the quality of the key frame that the SI will be made based on it, in low bit rates large volume of them will be requested and send because of low quality of key frames while they are not so effective for levels of perceived quality.

In Fig. 4, the impact of this issue is observed in both "Foreman" and "Hall monitor" (at low bit rate coding). As it is shown, even though generally the bit rate has been increased, in some cases the reduction in receiving quality is observed. For example, in Foreman sequence with bit rate encoding $109 \mathrm{kbps}$ two different levels of quality will be received. The higher quality is related to the case that video coding has done in higher rates and more details (Quantization Parameter $=44$ ) from beginning and size and quality of key frames are higher in it and lower quality is resulting by video coded with less detail $(\mathrm{QP}=49)$ that low quality of key frames in it cause of absence of appropriate SI and more need of decoder to the parity bits for WZ frame reconstruction. At this point, frame-by-frame bit rate and PSNR curves for Foreman has shown in In Fig. 5 and Fig. 6, that pair of indices in them are belong to WZ frames.

So, we can conclude that in DVC, quality of video frames and bit rate of WZ frames depend on bit rate and quality of key frames and generally, bit performance in key frame is much higher than bit performance in WZ frames; hence, considering this issue is necessary in selecting the encoding parameters.

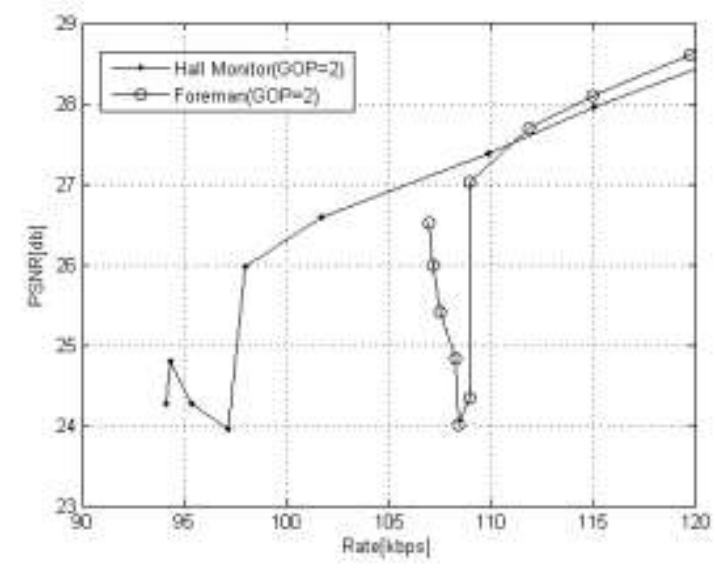

Fig. 4. Rate-Distortion in low bitrate encoding

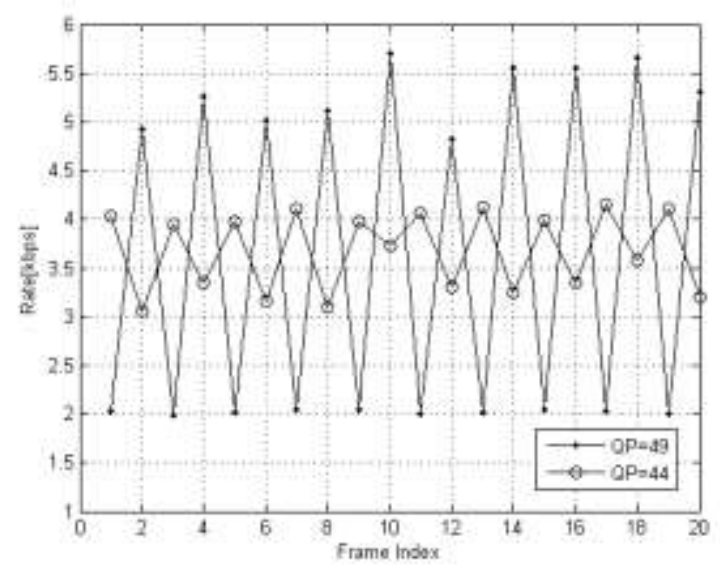

Fig. 5. two Frame-by-Frame bitrate pattern in 109 kbps encoding for the Foreman sequence 


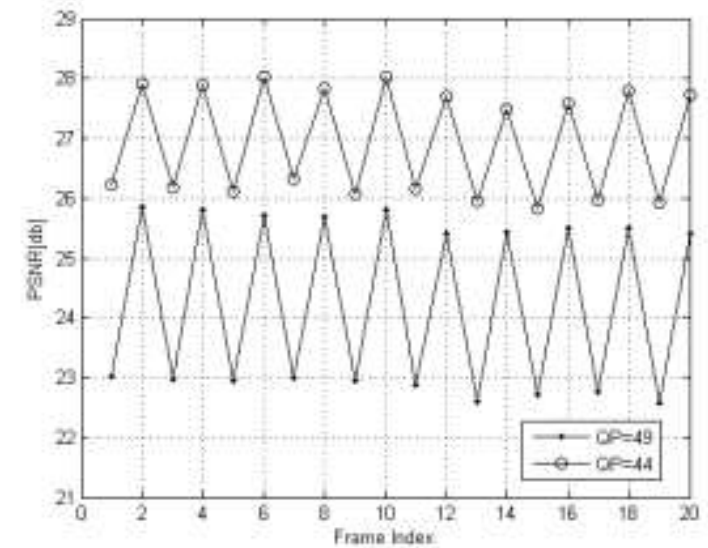

Fig. 6. two Frame-by-Frame PSNR pattern in 109 kbps encoding for the Foreman sequence

In Fig. 7, the relation between bit rate and receiving quality for both types of frames in Foreman is shown, separately. As it can be seen, increasing the bit rate and consequently increasing the receive quality in key frame will reduce the bit rate but increase the quality of $\mathrm{WZ}$ frames.

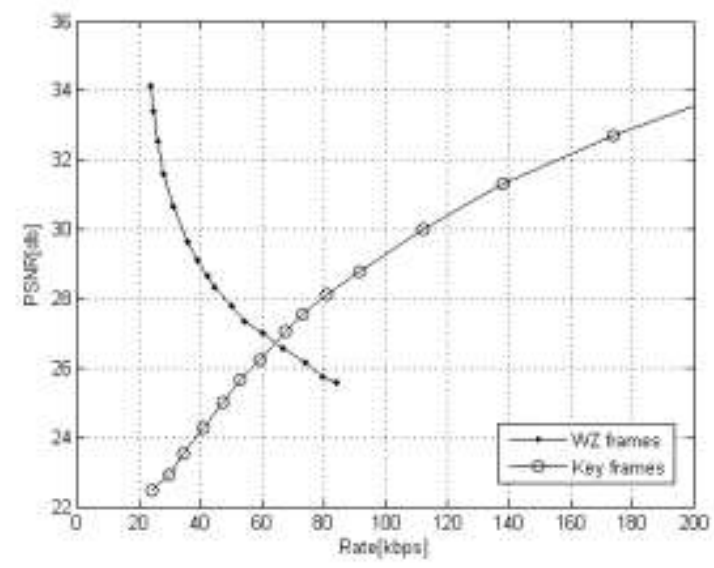

Fig. 7. Rate-Distortion in key and wyner-ziv frames

Also independent coding of frames and the channel feedback in this architecture cause that in low bit rate, the quality of WZ frames differs from the receive quality of the key frames due to getting more bits for the reconstruction. Even though it positively affects the average PSNR, it negatively affects the experimental quality of users because of the lack of uniformity frame's quality. We can overcome this issue by using a good approximation for the size of Wyner-Ziv frames that is proportional to the quality of the key frame but after transmission on erroneous wireless channel it will happen again, even more vigorously. For this reason, having the exact knowledge about this coding behavior in erroneous condition is necessary for confronting and controlling the error. In the next section of this research, the coding behavior in erroneous condition is investigated more realistically using simulation and applying a bit-level error model.

\section{ERROR RESILIENCE PERFORMANCE ANALYSIS}

Channel error commonly occurs in wireless networks. It is more common in WMSNs due to the low power transmitters [18]. In addition, to the video data, the data compression within frames and breaking them to smaller packets result in a packet loss may have adverse effect on the reconstruction of a GOP. But as previously stated, by frames independent coding, DVC is safe and behaves somewhat differently when encounters with channel error.

In this part of the study, we analyze the behavior of the DVC against channel error and discover the appropriate strategies to deal with it through the stimulation analysis. Since RF band is used in wireless sensor networks to transfer data and its error characteristics are not uniform over time, and given that, the video frame lengths are variable, so after packetizing, the packets will not have the same lengths, using packet-based error model will not provide valid results. Here, we use Gilbert-Elliot twostate Markov model which works at the bit level [19] to model the channel behavior. Researchers show that this model estimates the error characteristics in the wireless channel well $[20,21]$. Therefore it has been widely used in the literature regarding WSN [21, 22].

This model has two states named Good and Bad, to illustrate states with light and heavy bit error rate (BER) respectively. In Good state the channel has bit error probability $E_{g}$ with long intervals and in Bad state the channel has bit error probability $E_{b}$ with short intervals where, $E_{b}>>E_{g}$.

The state diagram of this model is shown in Fig. 8. In this state diagram, the values of $E_{g}$ and $E_{b}$ and the $2 \times 2$ stochastic transition matrix provide complete specification of this two-state Markov chain. To determine the probability of state transition, values $P_{B B}$ and $P_{G G}$ are required that are probability of transmit to Good or Bad states, given that current state is that mode. These values are based on actual experiences presented in several papers $[23,24]$.

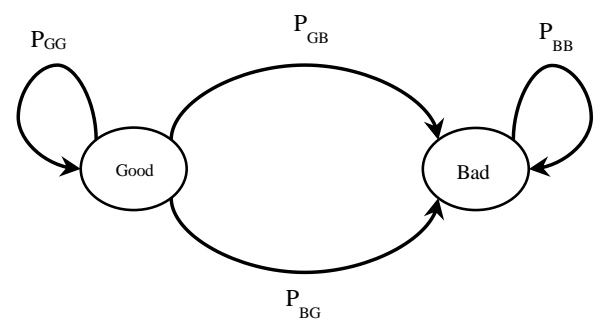

Fig. 8. two states Markov Chain of Gillbert-Elliot erasure channel model

In this model, the mean sojourn time in each of these states (duration of staying in a state) will be equal

$$
\begin{aligned}
T G & =1 / 1-P G G \\
T B & =1 / 1-P B B
\end{aligned}
$$

Then the mean bit error rate in this model can be calculated by (3): 


$$
\text { Average }(B E R)=T_{G} E_{g}+T_{B} E_{b}
$$

That reflects the impact of the transition probabilities between states on the average BER.

Using this model also known as the Gilbert-Elliot Erasure channel, due to burst errors model, preserves the independence of the frames packetizing method from channel model and yields more realistic results.

\section{A. simulation setup}

In order to measure the error resiliency of DVC and discover appropriate strategies to deal with channel error in it, Network Simulator (2) [25] and video quality measurement tool set called EvalVid [26] have been used. In the simulation scenario, a wireless sensor network within 200x200 meter with 50 wireless nodes capable of collecting, coding, and sending a live video to sink destination has been assumed. Physical characteristics that are based on MicaZ multimedia sensors [27] are presented in Table 1.

Table 1. Simulation Parameters

\begin{tabular}{c|c}
\hline Parameters & Value \\
\hline Channel Band with & $250 \mathrm{kbps}$ \\
Transmission Range & $40 \mathrm{~m}$ \\
Transmit Power & $52.2 \mathrm{mw}$ \\
Buffer Length & 100 \\
Packet Size & 200 Byte \\
\hline
\end{tabular}

In each simulation run, a pair of sender and receiver is selected randomly from the nodes in which the sender captures, encode with DVC and sends video sequences by taking random hops to the destination through the AODV routing protocol [28]. We have used a protocol based on a CSMA to manage medium access control.

Here, both the Foreman and Hall monitor sequences have been coded by DISCOVER in GOP Size $=2$ with $15 \mathrm{fps}$ in which the key frames are generated by H.264/AVC codec and are broken into packets of 200 bytes and in case of a packet loss, standard error concealment methods in H.264 are used to compensate it in the related frame. Additionally, WZ frames are grouped and transmitted in packets by using the approach presented in [11] and if they are lost due to errors, they will be requested and retransmitted through the feedback channel. The presented results are mean of 5 repetitions of simulation runs.

\section{B. simulation result}

Independent frames coding and feedback channel in DVC are the two reasons causing different behavior of this coding against channel errors. Admittedly receiving more bits from feedback channel will increase energy consumption and delay that both are important factors affecting the quality of service received from transmission framework in wireless sensor networks [29]. But since the purpose of this study is just to investigate the behavior of the codec against channel error, so our hypothesis is receiving WZ bits after one or several times of sending, without considering retransmission in bit rate.
Fig. 9 and Fig. 10 show decreasing pattern of PSNR with increasing bit error rate for two sequences.

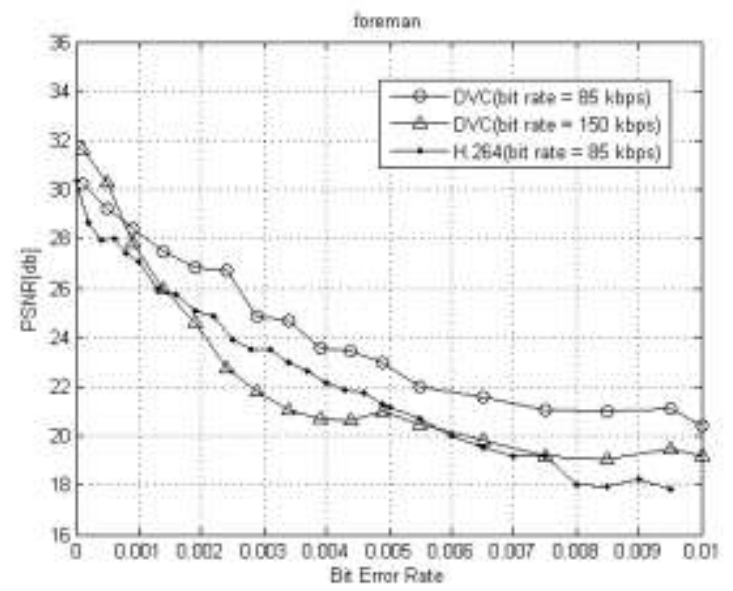

Fig. 9. PSNR vs. BER for the Foreman sequence in the two bitrate encoding

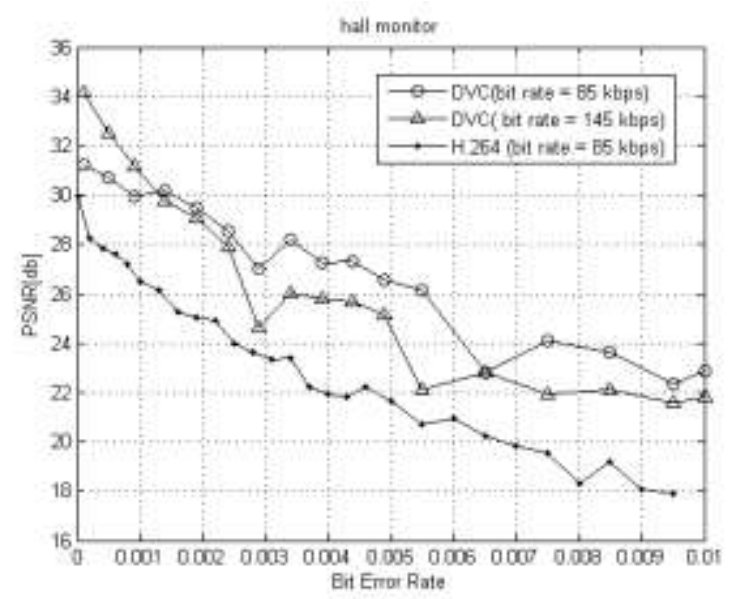

Fig. 10. PSNR vs. BER for the Hall monitor sequence in the two bitrate encoding

In these curves, further reduction in the average PSNR for H.264 video coded compared with coded video with DVC is evident. Although due to the lack of such channels in H.264 coding, it is not a fair comparison, but it shows the effect of feedback channel in DVC.

It is also observed for DVC that the receiving quality in video that encoded at high bit rates in different BERs is little away from the quality of video that encoded at lower bit rates and for Foreman in some cases the values are lower. Its main reason is that the key frames are more subjected to error that has been sent without any protection and they are more intensive at high bit rates. In fact, it highlights the impact and importance of the key frames and the need to protect them, which leads to an increase in receiving quality and it also reduces the number of request from feedback channel.

Another point worth noting according to these figures is further reducing PSNR with increasing the probability of error in Foreman to Hall monitor that indicates the effect of application type and his motion characteristics on behavior of this coding method against channel error. In fact, high motion of pictures in the Foreman sequence 
and the failure of the key frames that side information made based on them cause to form an inappropriate SI in the decoder side; thus, the number of requests from the feedback channel increases and the quality of Wyner-Ziv frames reduces. So, for DVC coding, when choosing encoding parameters and error control, we must pay more attention to this issue. Since independent encoding of frames in DVC causes independent behavior of the frames encountering the channel error, quality loss caused by the increase in bit error rate is reported separately for two kinds of frames in Fig. 11 and Fig. 12 where WZ frames is less affected due to the feedback channel and requiring more parity bits when does not able to reconstructing the frame. As it was said earlier, although it would have a positive impact on the average PSNR, it will have a disastrous impact on the experimental quality of the user.

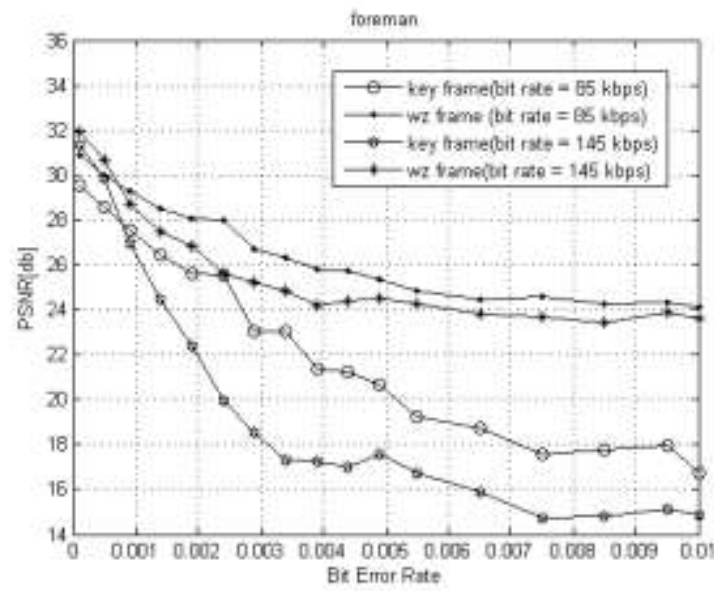

Fig. 11. frame-by-frame PSNR vs. BER for the Foreman sequence in the two bitrate encoding

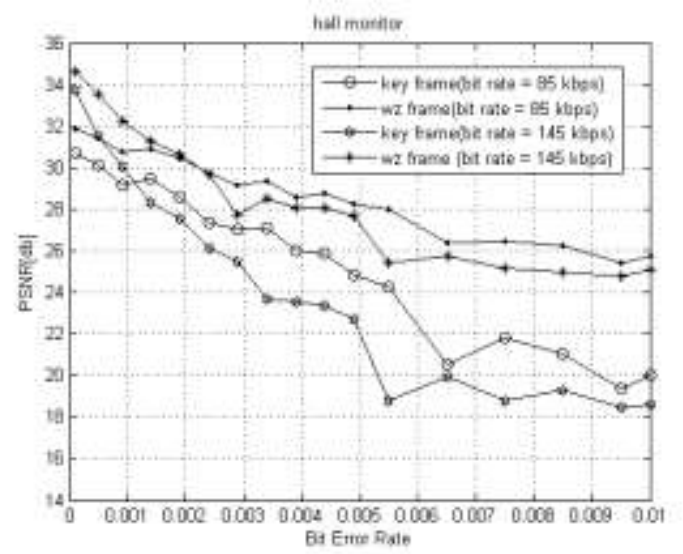

Fig. 12. frame-by-frame PSNR vs. BER for the Foreman sequence in the two bitrate encoding

The impact of this issue can be seen on five consecutive frames in both video sequences in the Fig. 13 and Fig. 14. According to Table 2, which presents the features of these five frames in terms of size and PSNR, it can be seen that WZ frames with number 91 in Foreman and number 37 in Hall monitor that key frames before and after it has poor quality, have been able to reconstruct a better picture and get more PSNR by getting more bits from feedback channel. But this increase compared to the volume of received bits from the feedback channel, which requires energy and causes delay would be negligible. This problem can be largely overcome by protection the key frames. For example, in WZ frames numbers 93 and 39 that have been shown respectively in Fig. 13 and Fig. 14 , although only the key frame after them has good quality, due to the formation of appropriate SI with this frame in decoder, less receiving from feedback channels has been done and they have had better quality than the three previous frames.

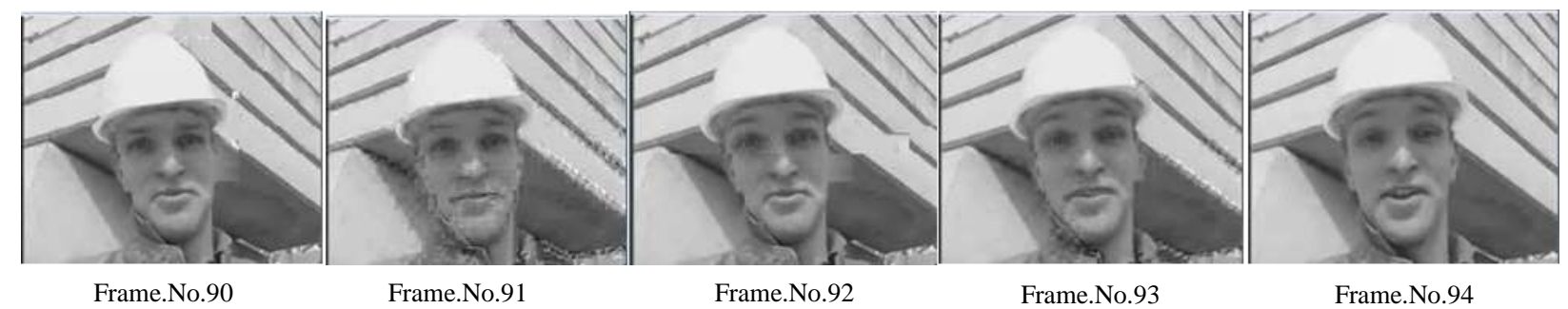

Fig. 13. five consecutive frames of the Foreman in BER=0.0015

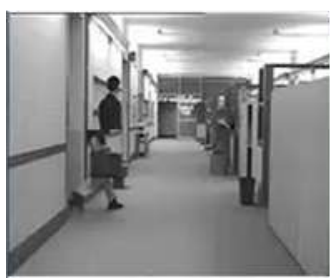

Frame.No.36

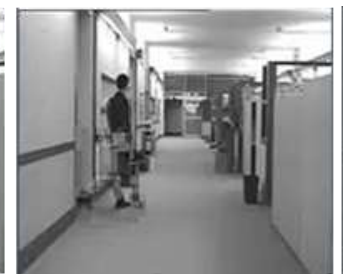

Frame.No.37

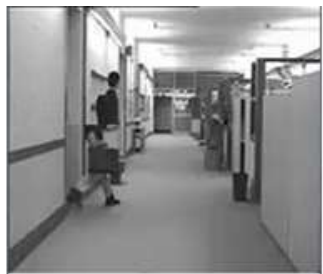

Frame.No.38

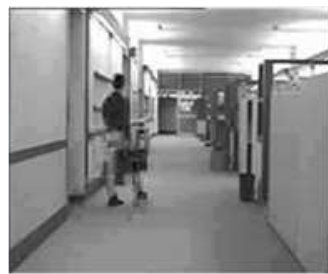

Frame.No.39

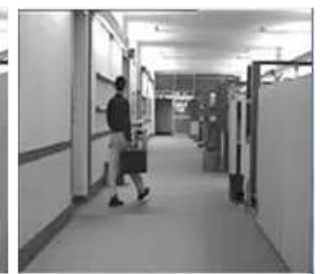

Frame.No.40

Fig. 14. five consecutive frames of the Hall monitor in $\mathrm{BER}=0.0015$

In the next section of this paper, by applying an appropriate error control on the key frames, we study and analyze its effects on PSNR and uniformity of its values between key and wyner-ziv frames through measures of 
objective and subjective assessments of video quality and also reduction of the amount of request from feedback channel.

Table 2. Rate and PSNR in 5 consecutive frames for the hall \& Foreman sequence

\begin{tabular}{|c|c|c|c|c|c|c|}
\hline \multirow{2}{*}{ Frame Type } & \multicolumn{3}{|c|}{ Foreman } & \multicolumn{3}{|c|}{ Hall monitor } \\
\hline & Frame.No & $\operatorname{Rate}(\mathrm{Kb})$ & $\operatorname{PSNR}(\mathrm{db})$ & Frame.No & $\operatorname{Rate}(\mathrm{Kb})$ & $\operatorname{PSNR}(\mathrm{db})$ \\
\hline $\mathrm{KY}$ & 90 & 16.58 & 20.37 & 36 & 17.8 & 26.12 \\
\hline WZ & 91 & 6.27 & 26.02 & 37 & 6.46 & 30.58 \\
\hline $\mathrm{KY}$ & 92 & 16.39 & 23.11 & 38 & 18.02 & 24.28 \\
\hline WZ & 93 & 3.82 & 28.74 & 39 & 2.43 & 31.54 \\
\hline KY & 94 & 16.55 & 35.35 & 40 & 18.23 & 34.81 \\
\hline
\end{tabular}

\section{Performance Assesment of Protected DVC By REED-SOLOMON FEC}

In the FEC error control techniques at the link layer, usually the sender adds some redundancy to the packets and by using them the receiver will be able to detect and correct the channel error that its value depends on the amount of redundancy. There are many methods for this type of error control. These methods are divided into two main categories: the Block Codes techniques such as Reed-Solomon or $\mathrm{BCH}$, and the Convolutional Codes techniques. In the first method, the data is divided into blocks that are then coded, while in the latter one data is processed bit by bit.

We have used Reed-Solomon error control in our analysis because as previously noted, in the RF channel we have encountered with burst errors. In this case, Block Code methods are more efficient and among them RS has lower power consumption than other methods [30].

In RS method, FEC encoder receives a block consists of $\mathrm{k}$ symbols and makes $\mathrm{n}$ symbols of channel. Here it is shown by $\mathrm{RS}$ (n, k) where $n \geq k$ and the decoder can be able to retrieve a block of up to $n-k / 2$ faulty symbol and it is not important that corrupted symbols have how much bit errors [26]. We have considered symbols as a byte and encode and send them by RS $(204,200)$.

\section{A. simulation result}

Comparing curves of the bit rate changes and receiving quality by increasing BER are plotted in Fig. 15 and Fig. 16 for Foreman in both high and low bit rates (similar result have been obtained for Hall monitor that due to lack of space are not shown here). Protective effects of the key frames via RS to maintain quality and avoid requiring feedback channel are well seen.

In fact, with adding redundancy to data and consuming more energy for decoding RS at each hop (Reed-Solomon encoder energy consumption is negligible) [28] we achieved a significant increase in quality of frames and there is no need to feedback channel and consequently reduction in the delay, that all of which remind us need to protect frames in DVC. By WZ frames protection through ARQ and reduce probability of losing these packets can achieve more reduction in number of request from the feedback channel.

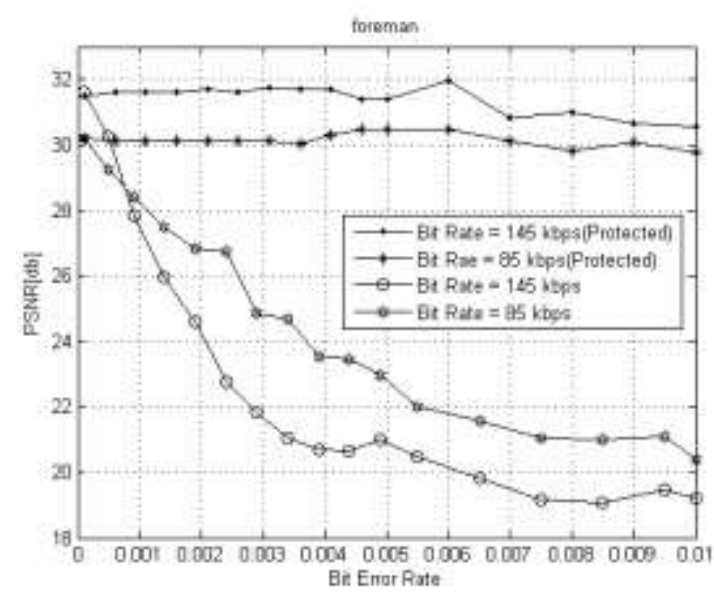

Fig. 15. PSNR vs. BER for the Foreman sequence in the two bitrate encoding and protected with $\mathrm{RS}(204,200)$

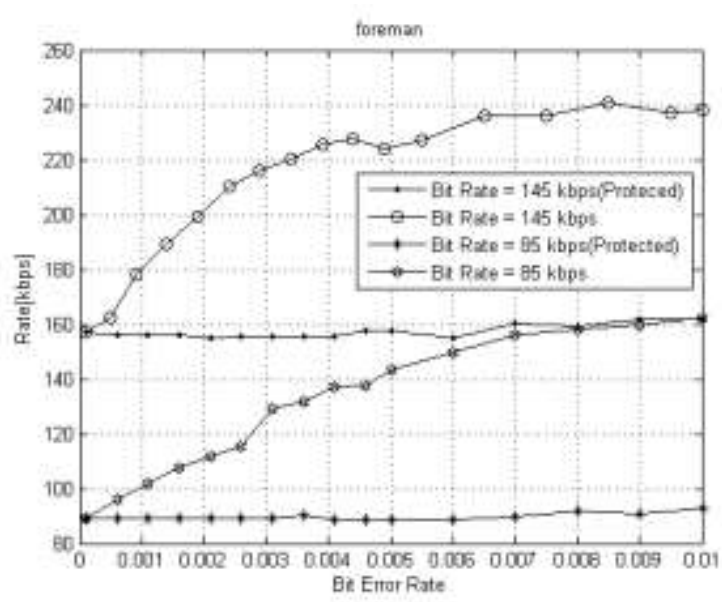

Fig. 16. Bitrate vs. BER for the Hall monitor sequence in the two bitrate encoding and protected with RS(204,200)

In Fig. 17 MOS criteria has been used to show the distribution of frames quality in protected and unprotected conditions. Mean Opinion Score is a subjective parameter for measuring the quality of experience (QoE) of a video in application-level that shows user experimental quality with a score ranging from 1 to 5 [26]. Here, PSNR values for each frame has been mapped to MOS based on Table 3, and percentage of frames in each area has been calculated. 


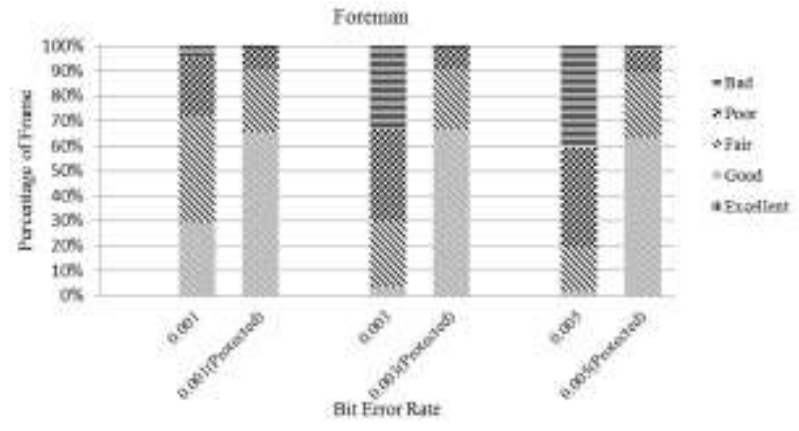

Fig. 17. Compare PSNR distribution in protected and unprotected Foreman sequence

Table 3. PSNR mapping to MOS

\begin{tabular}{cc}
\hline MOS & PSNR \\
\hline 5 (Excellent) & $>37$ \\
4 (Good) & $31-37$ \\
3 (Fair) & $25-31$ \\
2 (Poor) & $20-25$ \\
1 (Bad) & $<20$ \\
\hline
\end{tabular}

According to these figure it can be understood that the problem of quality fluctuating between the key and WZ frames described in Section 3, is largely removed, by protecting key frames in addition to a significant increase in the number of frames with Good quality. In Fig. 18, the values of PSNR for 150 subsequent frames have been depicted for the Foreman sequence that confirms this result. The reduction of the frequency and severity of such fluctuations occurrence can be seen clearly after applying the error control on the key frames.

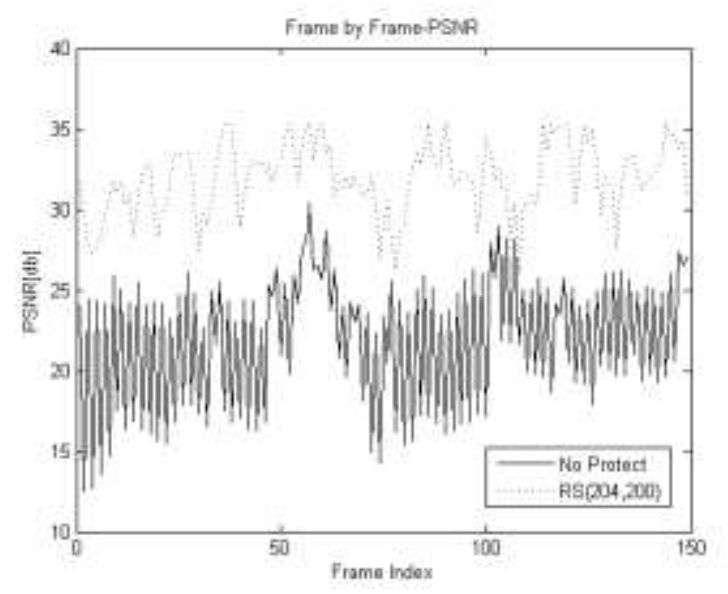

Fig. 18. frame-by-frame PSNR in Foreman

\section{CONCOLUSION}

In this study, according to the bit rate importance and error resiliency in video transmission over WMSN, we have studied and compared bit rate efficiency in DVC and its error resiliency. To do this, we have used twostate Gilbert-Elliot channel models to capture the bit level channel error. Based on the results, the performance of the bit rate in key frames is much higher than WZ frames.
In addition, despite frames independent coding, in this coding method the error in the key frames will increase $\mathrm{WZ}$ bit rate significantly. The increase of $\mathrm{WZ}$ bit rate somewhat compensates the distortion in this frames; however it causes fluctuations in quality of the key frames and reduction of QoE. In addition, receiving bits from feedback channel requires energy and it will increase the delay. Then the effect of protection of the key frames in reduction in bit rate have been shown by the Link Layer FEC (Reed-Solomon) and increasing the average PSNR as well as MOS, was shown in a separate analysis according to which we can largely overcome the problems by protection of the frame mentioned. Therefore, error control in DVC can be considered as a necessity that must be used by complete understanding the efficiency of various methods for error control. Performance analysis of error control methods and providing efficient methods for it considering the limitations in the WMSN and DVC coding features will be pursued in future work.

\section{REFERENCES}

[1] I. F. Akyildiz, T. Melodia, and K. R. Chowdury, "Wireless multimedia sensor networks: A survey," Wireless Communications, IEEE, vol. 14, pp. 32-39, 2007.

[2] I. F. Akyildiz, T. Melodia, and K. Chowdhury, "Wireless multimedia sensor networks: Applications and testbeds," Proc. IEEE, vol. 96, no. 10, pp.1588-1605, October 2008.

[3] F. Falah, L. Suriani Affendey,"Systematic Review and Classification on Video Surveillance Systems", IJITCS, vol.5, no.7, pp.87-102, 2013.

[4] B. Girod, A. M. Aaron, S. Rane, and D. Rebollo-Monedero, "Distributed Video Coding," Proceedings of the IEEE, vol. 93, pp. 71-83, 2005.

[5] R. Puri, A. Majumdar, P. Ishwar, K. Ramchandran, "Distributed video coding in wireless sensor networks," IEEE Signal Proc. Magazine, vol. 23, pp. 94-106, 2006.

[6] V. K. Kodavalla and P. G. K. Mohan, "Multi-view distributed video coding," in Devices, Circuits and Systems (ICDCS), 2012 International Conference on, 2012, pp. 614-618.

[7] L. Van Hoesel, T. Nieberg, W. Jian, and P. J. M. Havinga, "Prolonging the lifetime of wireless sensor networks by cross-layer interaction," Wireless Communications, IEEE, vol. 11, pp. 78-86, 2004.

[8] J. J. Ahmad, H. A. Khan, and S. A. Khayam, "Energy efficient video compression for wireless sensor networks," in Information Sciences and Systems, 2009. CISS 2009. 43rd Annual Conference on, 2009, pp. 629-634.

[9] D. Slepian and J. K. Wolf, "Noiseless coding of correlated information sources," Information Theory, IEEE Transactions on, vol. 19, pp. 471-480, 1973.

[10] A. D. Wyner and J. Ziv, "The rate-distortion function for source coding with side information at the decoder," Information Theory, IEEE Transactions on, vol. 22, pp. 110, 1976.

[11] C.Tonoli, M.Migliorati, and R.Leonardi, " Error resilience performance evalution of a distributed video codec, " in Proceedings of the International Picture Coding Symposium (PCS '07), vol. 6, pp. 14445-14451, Lisbon, Portugal, November 2007.

[12] A. Aaron, R. Zhang, and B. Girod, "Wyner-Ziv coding for motion video," in Proceedings of the 36th Asilomar 
Conference on Signals, Systems and Computers, Pacific Grove, Calif, USA, November 2002

[13] A. Aaron, S. D. Rane, E. Setton, and B. Girod, "Transform domain Wyner-Ziv codec for video," in Visual Communications and Image Processing, vol. 5308 of Proceedings of SPIE, pp. 520-528, San Jose, Calif ,USA, January 2004.

[14] R. Puri and K. Ramchandran, "PRISM: a new robust video coding architecture based on distributed compression principles," in Proceedings of the 40th Allerton Conference on Communication, Control and Computing, Allerton, Ill, USA, October 2002.

[15] R. Puri and K. Ramchandran, "PRISM: a new "reversed" multimedia coding paradigm," in Proceedings of International Conference on Image Processing (ICIP '03), vol. 1, pp. 617-620, Barcelona, Spain, September 2003.

[16] G. A. Shah, L. Weifa, and O. B. Akan, "Cross-Layer Framework for QoS Support in Wireless Multimedia Sensor Networks," IEEE Transactions on Multimedia, vol. 14, pp. 1442-1455, 2012.

[17] X. Artigas, J. Ascenso, M. Dalai, S. Klomp, D. Kubasov, and M. Ouaret, "The DISCOVER codec: architecture, techniques and evaluation," in Proceedings of the International Picture Coding Symposium (PCS '07), vol. 6, pp. 14496-14410, Lisbon, Portugal, November 2007.

[18] M. Roshanzadeh,S. Saqaeeyan,"Error Detection \& Correction in Wireless Sensor Networks By Using Residue Number Systems", IJCNIS, vol.4, no.2, pp.29-35, 2012

[19] M. Mushkin and I. Bar-David, "Capacity and coding for the gilbert-elliott channels," Information Theory, IEEE Transactions on, vol. 35, pp. 1277-1290, 1989.

[20] W. Hong-Shen and C. Pao-Chi, "On verifying the firstorder Markovian assumption for a Rayleigh fading channel model," Vehicular Technology, IEEE Transactions on, vol. 45, pp. 353-357, 1996.

[21] K. L. Blackard, T. S. Rappaport, and C. W. Bostian, "Radio frequency noise measurements and models for indoor wireless communications at $918 \mathrm{MHz}, 2.44 \mathrm{GHz}$, and 4.0 GHz," in Communications, 1991. ICC '91, Conference Record. IEEE International Conference on, 1991, pp. 28-32 vol.1.

[22] M. Y. Naderi, H. R. Rabiee, M. Khansari, and M. Salehi, "Error control for multimedia communications in wireless sensor networks: A comparative performance analysis," $\mathrm{Ad}$ Hoc Networks, vol. 10, pp. 1028-1042, 2012.

[23] J. Pierre Ebert and A. Willig, "A Gilbert-Elliot Error Model and the Efficient Use in Packet Level Simulation," Technical University Berlin, TKN Technical Report, TKN99-002, March 1999.

[24] D. Duchamp and N. F. Reynolds, "Measured performance of a wireless LAN," in Local Computer Networks, 1992. Proceedings., 17th Conference on, 1992, pp. 494-499.

[25] UC Berkeley, LBL, USC/ISI and Xerox PARC, The Network Simulator ns-2, The VINT Project.[Online] Available: http://www.isi.edu/nsnam/ns/.

[26] C. Ke, C. Shieh, W. Hwang, and A. Ziviani, "An evaluation framework for more realistic simulations of MPEG video transmission," J. Inf. Sci. Eng., vol. 440, no. 2, pp. 425-440, 2008.

[27] I. Akyildiz and M. Vuran, "Wireless Sensor Networks," John Wiley \& Sons, 2010.

[28] C. Perkins, E.M. Belding-Royer, S. Das, Ad hoc on demand distance vector (aodv) routing, IETF RFC 3561, 2003.

[29] L. Van Hoesel, T. Nieberg, W. Jian, and P. J. M. Havinga, "Prolonging the lifetime of wireless sensor networks by cross-layer interaction," Wireless Communications, IEEE, vol. 11, pp. 78-86, 2004.

[30] S. Lin, D.J. Costello, Error Control Coding: Fundamentals and Applications, Prentice Hall, 1983.

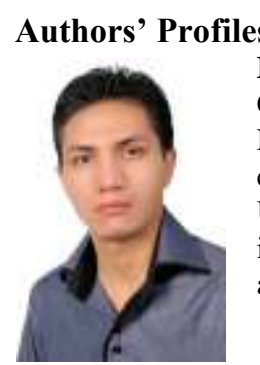

Mortaza Nikzad received the BS degrees in Computer engineering from the Payam-eNoor University, Iran in 2011. He is now completing the MSC degree at the University of Isfahan, Iran. His research interests include multimedia communication and wireless sensor networks.

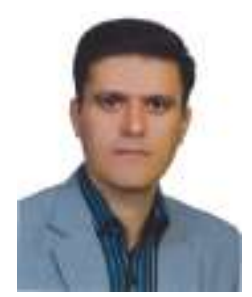

Ali Bohlooli received the BS and MS degrees in Computer engineering (with honors) from the department of Electrical \& Computer Engineering, Isfahan University of Technology, Iran in 2001 and 2003, respectively. He received his Ph.D. degree from the University of Isfahan, Iran in 2011. Now he is an assistant professor at department of Computer Engineering, University of Isfahan, Iran. His research interests include wireless networks and network modeling.

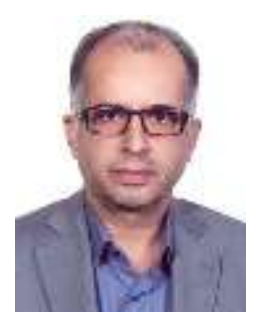

Kamal Jamshidi received the MS and $\mathrm{PhD}$ degrees in electrical engineering from Anna University of India in 1990 and I.I.T University of India in 2003, respectively. $\mathrm{He}$ currently is an associate professor at department of Computer Engineering, University of Isfahan, Iran. His research interests include wireless sensor network as well as fuzzy systems and microprocessor

based systems.

How to cite this paper: Mortaza Nikzad, Ali Bohlooli, Kamal Jamshidi,"Video Quality Analysis of Distributed Video Coding in Wireless Multimedia Sensor Networks", International Journal of Information Technology and Computer Science(IJITCS), vol.7, no.1, pp.12-20, 2015. DOI: 10.5815/ijitcs.2015.01.02 